\title{
Effects of Minor Ginsenosides, Ginsenoside Metabolites, and Ginsenoside Epimers on the Growth of Caenorhabditis elegans
}

\author{
Joon-Hee Lee ${ }^{1 \#}$, Ji-Yun Ahn ${ }^{2 \#}$, Tae-Joon Shin ${ }^{3 \#}$, Sun-Hye Choi ${ }^{3}$, Byung-Hwan Lee ${ }^{3}$, Sung-Hee \\ Hwang $^{3}$, Jiyeon Kang ${ }^{3}$, Hyeon-Joong Kim ${ }^{3}$, Chan-Woo Park ${ }^{3}$, and Seung-Yeol Nah ${ }^{3^{*}}$ \\ ${ }^{1}$ Department of Physical Therapy, Daebul University, Yeongam 526-702, Korea \\ ${ }^{2}$ Food Function Research Division, Korea Food Research Center, Seongnam 463-746, Korea \\ ${ }^{3}$ Ginsentology Research Laboratory and Department of Physiology, Konkuk University College of Veterinary Medicine, Seoul \\ 143-701, Korea
}

In the previous report, we have demonstrated that ginsenoside Rc, one of major ginsenosides, is a major component for the restoration for normal growth of worms in cholesterol-deprived medium. In the present study, we further investigated the roles of minor ginsenosides, such as ginsenoside $\mathrm{Rh}_{1}$ and $\mathrm{Rh}_{2}$, ginsenoside metabolites such as compound $\mathrm{K}(\mathrm{CK})$, protopanaxadiol (PPD), and protopanaxatriol (PPT) and ginsenoside epimers such as $20(R)$ - and 20(S)-ginsenoside $\mathrm{Rg}_{3}$ in cholesterol-deprived medium. We found that ginsenoside $\mathrm{Rh}_{1}$ almost restored normal growth of worms in cholesterol-deprived medium in F1 generation. However, supplement of ginsenoside $\mathrm{Rh}_{2}$ caused a suppression of worm growths in cholesterol-deprived medium. In addition, CK and PPD also slightly restored normal growth of worms in cholesterol-deprived medium but PPT not. In experiments using ginsenoside epimers, supplement of 20(S)- but not 20(R)-ginsenoside $\mathrm{Rg}_{3}$ in cholesterol-deprived medium also almost restored worm growth. These results indicate that the absence or presence of carbohydrate component at backbone of ginsenoside, the number of carbohydrate attached at carbon-3, and the position of hydroxyl group at carbon-20 of ginsenoside might plays important roles in restoration of worm growth in cholesterol-deprived medium.

Keywords: Panax ginseng, Caenorhabditis elegans, Ginsenoside metabolites, Ginsenoside epimers, Growth

\section{INTRODUCTION}

Ginseng, the root of Panax ginseng Meyer, has been used as a representative tonic for two thousand years in the Far East countries like Korea, China, and Japan. Now, ginseng is one of the most famous and precious herbal medicines consumed around the world [1]. Although ginseng exhibits multiple pharmacological actions in vitro or in vivo studies, its mechanisms on various efficacies are still elusive. Recent accumulating evidences show that ginseng saponins (or ginsenosides) are the main active

(cc) This is an Open Access article distributed under the terms of the Creative Commons Attribution Non-Commercial License (http://creativecommons.org/licenses/by-nc/3.0/) which permits unrestricted non-commercial use, distribution, and reproduction in any medium, provided the original work is properly cited. ingredients of ginseng (Fig. 1). Ginseng root contains $3 \%$ to $4 \%$ of ginseng saponins. Ginseng saponins are especially abundant in fine roots rather than main body of ginseng root. Ginseng saponins are one of glycoside saponins and one of the derivatives of triterpenoid dammarane consisting of thirty carbon atoms. Each ginsenoside has a common hydrophobic four ring cholesterol-like backbone structure with sugar moieties attached. About 30 different types of ginseng saponins have been isolated

\footnotetext{
Received 08 Jun. 2011, Revised 14 Sep. 2011, Accepted 15 Sep. 2011

${ }^{\#}$ These authors contributed equally to this work.

*Corresponding author

E-mail: synah@konkuk.ac.kr

Tel: +82-2-450-4154, Fax: +82-2-450-3037
} 


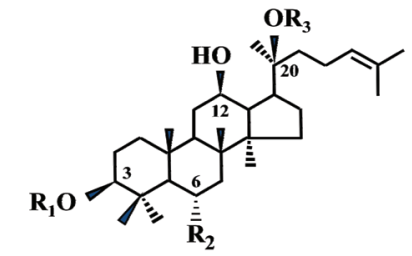

\begin{tabular}{|c|c|c|c|}
\hline Ginsenosides & $\mathbf{R 1}$ & $\mathbf{R 2}$ & $\mathbf{R 3}$ \\
\hline $\mathbf{R} \mathbf{b}_{1}$ & - Glc $_{2}$-Glc & $-\mathrm{H}$ & $-\mathbf{G l c}_{6}-\mathbf{G l c}$ \\
\hline Re & - Glc $_{2}$-Glc & $-\mathbf{H}$ & - Glc $_{6}$-Ara(fur) \\
\hline $\mathbf{R} \mathbf{h}_{1}$ & $-\mathrm{OH}$ & -GIc & $-\mathbf{H}$ \\
\hline $\mathbf{R h}_{2}$ & -Glc & $-\mathbf{H}$ & $-\mathbf{H}$ \\
\hline $\mathbf{R g}_{3}$ & -Glc -Glc $^{-}$ & $-\mathbf{H}$ & $-\mathrm{H}$ \\
\hline PPD & $-\mathrm{H}$ & $-\mathbf{H}$ & $-H$ \\
\hline PPT & $-\mathbf{H}$ & $-\mathrm{OH}$ & $-\mathbf{H}$ \\
\hline CK & $-\mathbf{H}$ & $-\mathbf{H}$ & -GIc \\
\hline
\end{tabular}

Fig. 1. Structures of the representative ginsenosides and ginsenoside metabolites. They differ at three side chains attached the common steroid ring. Subscripts indicate the carbon in the glucose ring that links the two carbohydrates. Glc, glucopyranoside; Ara(fur), arabinofuranose; PPD, protopanaxadiol; PPT, protopanaxatriol; CK, compound $\mathrm{K}$.

and identified from the root of $P$. ginseng (Fig. 1).

Caenorhabditis elegans is one of nematode species and is one of free living animals under the earth rather than is parasitic in host animals or plants. C. elegans takes its food from dead animals or plants. Since C. elegans has short life cycle and large amounts of worms can be easily grown for biochemical assays or genetic studies in the laboratory, the organism is one of the wellestablished genetic models and its development processes have well characterized. Interestingly, C. elegans cannot synthesize sterols unlike other animals and requires dietary sterol, which is usually supplied as cholesterol in the laboratory [2,3]. Although the functions of sterols in C. elegans have not been fully characterized, in vitro sterol deprivation results in decreased fertility, delayed development, and short life span throughout the first(F1), second (F2), and third (F3) generations $[3,4]$.

On the other hand, some species of parasitic nematodes are found in wild and cultivated ginseng root. For example, Pratylenchus subpenetran is one of main parasitic animals and causes damage to ginseng roots [5]. It penetrates into fine roots or outer spaces of ginseng root from soil and makes a lot of small humps in fine roots, because they die if they are exposed to air. Its parasitic actions in fine roots of ginseng interfere to absorb nutrients from soil. It is known that if it is not treated with anti-nematode agents, it causes to severe damages on normal development of ginseng during young period, resulting in delay of the growth and further make gin- seng fragile to other microbial infections. Although these observations suggest a possibility that ginseng roots might provide suitable environment(s) for survival of the parasitic nematodes, little is known on effects of ginseng saponins or individual ginsenosides as main ingredients of ginseng on the growth, development, and life span of nematodes.

In the previous report, we have shown that supplements of ginseng total saponins (GTS) fraction and ginsenoside $\mathrm{Rb}_{1}$ and $\mathrm{Rc}$ restored normal growth of C. elegans grown in cholesterol-deprived-medium [6]. These results show a possibility that the individual ginsenosides might have differential effects on growth of $C$. elegans. On the other hand, recent works have shown that ginsenosides administered via the oral route may pass into the large intestine without being decomposed by either gastric juices or digestive enzymes [7]. Ginsenoside $\mathrm{Rh}_{1}$ is metabolized into protopanaxatriol (PPT) by intestinal microorganisms, whereas ginsenoside $\mathrm{Rh}_{2}$ is metabolized into protopanaxadiol (PPD). They possess only the backbone structure of ginsenosides without any carbohydrate component [7] (Fig. 1). Some of these ginsenoside metabolites have shown to have anticancer activity [7]. We have also shown that ginsenoside epimers have differential effects on voltage-gated ion channel regulations [8]. For example, 20(S)-ginsenoside $\mathrm{Rg}_{3}$ but not 20(R)ginsenoside $\mathrm{Rg}_{3}$ inhibited voltage-dependent $\mathrm{Ca}^{+}, \mathrm{K}^{+}$, and $\mathrm{Na}^{+}$channel currents [8].

In the present study, we examined whether supplements of minor ginsenosides, ginsenoside metabolites, or ginsenoside epimers show any effects on the growth of C. elegans grown in cholesterol-deprived-medium. We found that minor ginsenoside, ginsenoside metabolites, and ginsenoside epimers showed differential effects on worm growth in cholesterol-depleted medium, depending on the number of carbohydrate attached at carbon- 3 and on the position of hydroxyl group at carbon-20 of ginsenoside $\mathrm{Rg}_{3}$. Thus, it appears that the number of carbohydrate attached at carbon-3 and the position of hydroxyl group at carbon-20 of ginsenoside $\mathrm{Rg}_{3}$ might contribute to the restoration of worm growth in cholesterol-deprived medium.

\section{MATERIALS AND METHODS}

\section{Materials}

Electrophoresis grade agarose was obtained from Becton, Dickinson and Company (Sparks, MD, USA) and peptone was obtained from Amresco (Solon, OH, USA). Cholesterol and all other analytical agents were obtained 
from Sigma (St. Louis, MO, USA). Cholesterol stock solution for cholesterol treatment group was prepared at $5 \mathrm{mg} / \mathrm{mL}$ of ethanol. The final ethanol concentration was $0.01 \%$. Minor ginsenosides, ginsenoside metabolites, and ginsenoside epimers, isolated according to the method of Tanaka et al. [9] and Shibata et al. [10], respectively, was kindly provided by the Korea Ginseng Corporation (Seoul, Korea). After chromatography, we confirmed no existence of phytosterols by HPLC. Fig. 1 shows the structures of ginsenoside metabolites and ginsenoside epimers.

\section{Media and Caenorhabditis elegans growth}

The nematodes were grown and maintained on NGM agarose plates $(3 \mathrm{~g} / \mathrm{L} \mathrm{NaCl}, 2.5 \mathrm{~g} / \mathrm{L}$ peptone, $5 \mathrm{mg} / \mathrm{L}$ cholesterol, $1 \mathrm{mM} \mathrm{CaCl}, 1 \mathrm{mM} \mathrm{MgSO} 4,25 \mathrm{mM} \mathrm{KH}_{2} \mathrm{PO}_{4}$, $\mathrm{pH} 6.0$, and $17 \mathrm{~g} / \mathrm{L}$ agar) with the Escherichia coli OP50 strain in an incubator at $20^{\circ} \mathrm{C}$ [11]. To obtain cholesterolfree conditions, agar was replaced by agarose, which was extracted three times with chloroform [12]. We also extracted peptone with ether in a large beaker in a fume hood [3]. For this, the peptone powder was mixed with an excess volume of ether, allowed to settle, decanted, and the process was repeated twice more. The extracted peptone was allowed to dry overnight in the hood to remove the remaining ether [3]. E. coli strain OP50 was also grown directly in this sterol-free medium. Wildtype N2 C. elegans (Bristol type) was provided by the Caenorhabditis Genetics Center and was maintained according to the methods of Brenner [11]. For definition of various generations of worms, we first cultured worms (Po, $n=10-20)$ in each treatment group such as cholesterol $(5 \mu \mathrm{g} / \mathrm{mL})$-fed, cholesterol-deprived, minor ginsenosides+cholesterol-deprived, ginsenoside metabolites + cholesterol-deprived, or ginsenoside epimers + cholesterol-deprived group. When eggs from Po animals grown in each treatment were again placed on various treatment group plates as mentioned above, the resulting animals are referred to as F1 generation. When eggs from F1 animals were grown in various treatment group plates, the resulting animals are referred to as F2 generation.

Table 1. Effects of minor ginsenosides, ginsenoside metabolites, and ginsenoside epimers on growth rate of Caenorhabditis elegans grown in cholesterol-deprived medium

\begin{tabular}{|c|c|c|c|}
\hline & \multicolumn{3}{|c|}{ Growth rate (h) (egg to adult) } \\
\hline & L4 & Young adult & Adult \\
\hline \multicolumn{4}{|l|}{ First generation } \\
\hline+ Chol & $65 \pm 0.2$ & $76 \pm 0.2$ & $96 \pm 0.2$ \\
\hline -Chol & $74 \pm 0.1^{* *}$ & $92 \pm 0.1^{* * * *}$ & $115 \pm 0.1^{* * *}$ \\
\hline$-\mathrm{Chol}+\mathrm{Rh}_{1}$ & $65 \pm 0.1$ & $89 \pm 0.1^{* * * *}$ & $96 \pm 0.1$ \\
\hline$-\mathrm{Chol}+\mathrm{Rh}_{2}$ & $72 \pm 0.2^{* * *}$ & $96 \pm 0.2^{* *}$ & $145 \pm 0.2^{* * * *}$ \\
\hline$-\mathrm{Chol}+\mathrm{CK}$ & $73 \pm 0.2^{* * *}$ & $114 \pm 0.1^{* * * *}$ & $139 \pm 0.1^{* * * *}$ \\
\hline$-\mathrm{Chol}+\mathrm{PPD}$ & $80 \pm 0.2^{* *}$ & $112 \pm 0.2^{* * * *}$ & $136 \pm 0.2^{* * * *}$ \\
\hline$-\mathrm{Chol}+\mathrm{PPT}$ & $94 \pm 0.2^{* * *}$ & $118 \pm 0.2^{* * * *}$ & $142 \pm 0.2^{* * * *}$ \\
\hline- Chol $+20(R) \operatorname{Rg}_{3}$ & $73 \pm 0.1^{* *}$ & $95 \pm 0.1^{* * * *}$ & $119 \pm 0.1^{* * * *}$ \\
\hline$-\mathrm{Chol}+20(S) \mathrm{Rg}_{3}$ & $69 \pm 0.1^{* * *}$ & $80 \pm 0.1$ & $96 \pm 0.2$ \\
\hline \multicolumn{4}{|l|}{ Second generation } \\
\hline+ Chol & $65 \pm 0.1$ & $76 \pm 0.2$ & $96 \pm 0.2$ \\
\hline -Chol & $76 \pm 0.1^{* *}$ & $100 \pm 0.1^{* * * *}$ & $123 \pm 0.1^{* * * *}$ \\
\hline$-\mathrm{Chol}+\mathrm{Rh}_{1}$ & $72 \pm 0.1^{* * *}$ & $95 \pm 0.1^{* *}$ & $120 \pm 0.1^{* * * *}$ \\
\hline$-\mathrm{Chol}+\mathrm{Rh}_{2}$ & $72 \pm 0.2^{* * *}$ & $95 \pm 0.2^{* * *}$ & $144 \pm 0.2^{* * *}$ \\
\hline$-\mathrm{Chol}+\mathrm{CK}$ & $90 \pm 0.2^{* *}$ & $119 \pm 0.1^{* * * *}$ & $144 \pm 0.1^{* * * *}$ \\
\hline$-\mathrm{Chol}+\mathrm{PPD}$ & $95 \pm 0.2^{* * *}$ & $120 \pm 0.2^{* * * *}$ & $144 \pm 0.2^{* * * *}$ \\
\hline- Chol + PPT & $95 \pm 0.2^{* * *}$ & $120 \pm 0.1^{* * *}$ & $144 \pm 0.1^{* * *}$ \\
\hline$-\mathrm{Chol}+20(R) \mathrm{Rg}_{3}$ & $73 \pm 0.1^{* * *}$ & $95 \pm 0.2^{* * * *}$ & $120 \pm 0.1^{* * * *}$ \\
\hline$-\mathrm{Chol}+20(S) \mathrm{Rg}_{3}$ & $69 \pm 0.2$ & $80 \pm 0.1$ & $99 \pm 0.2$ \\
\hline
\end{tabular}

Data are mean \pm SE ( $n=20$ for each stage).

L4, larvae 4; Chol, cholesterol; CK, compound K; PPD, protopanaxadiol; PPT, protopanaxatriol.

$" p<0.01, " ' p<0.001$, significantly different from cholesterol-fed group. 


\section{Measurement of growth rate and worm length}

Larvae 4 (L4) hermaphrodites $(n=20-30)$ grown in cholesterol-fed, cholesterol-deprived, minor ginsenosides + cholesterol-deprived, ginsenoside metabolites + cholesterol-deprived, or ginsenoside epimers+cholesterol-deprived group were individually cloned onto agarose plates at $20^{\circ} \mathrm{C}$. Growth rate of worms on each agarose plate was determined by observation of characteristic stage-specific morphology every $24 \mathrm{~h}$ until the end of the growth. We put individual worm at a specific stage on an agarose pad and measured the length of it. We also analyzed growth rate of F1 and F2 progenies with the same procedure as described above.

\section{RESULTS}

\section{Effects of minor ginsenosides on worm growth in cholesterol-deprived medium}

Worms grown in NGM agarose plates at $20^{\circ} \mathrm{C}$ were divided into three groups: cholesterol $(5 \mu \mathrm{g} / \mathrm{mL})$-fed, cholesterol-deprived, or cholesterol-deprived but supplemented with minor ginsenosides such as ginsenoside $\mathrm{Rh}_{1}$ or $\mathrm{Rh}_{2}(300 \mu \mathrm{M}$, each). We first examined the growth rate in cholesterol-deprived or fed-medium. As shown in Table 1 and Fig. 2A, in F1 generation the growth rate (i.e., the time to reach at adult stage from egg) of worms grown in cholesterol-deprived medium were significantly retarded by 9,16 , and $19 \mathrm{~h}$ at L4, young adult, and adult
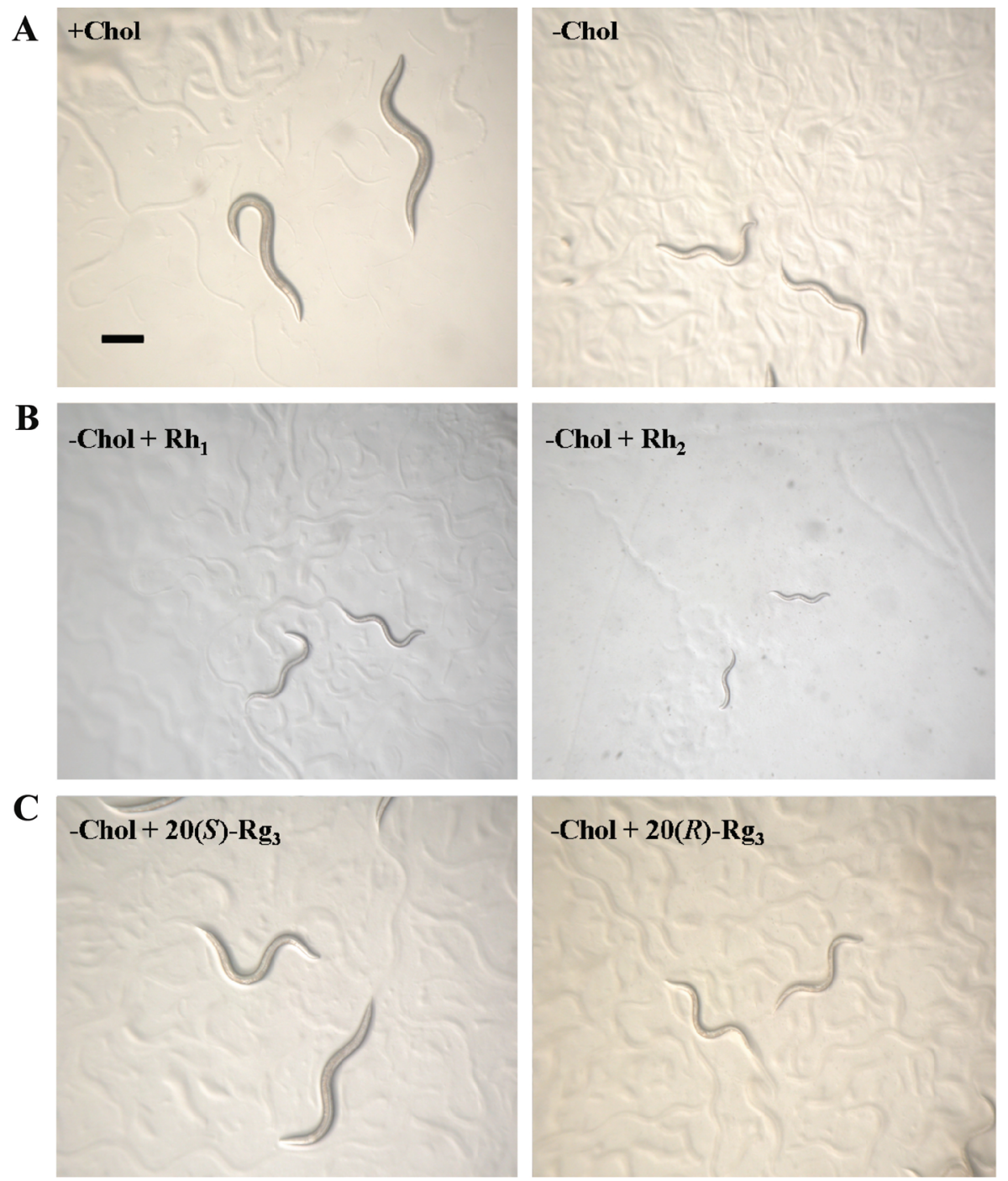

Fig. 2. Eggs laid by first generation animals were placed on plates containing the following concentrations: $(A)$ cholesterol $5 \mathrm{mg} / \mathrm{mL}(+\mathrm{Chol})$,

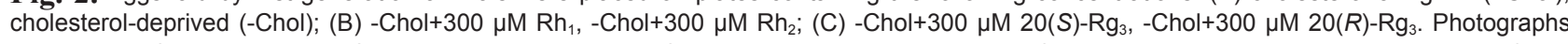
were taken after $72 \mathrm{~h}$ growth of second generations at $20^{\circ} \mathrm{C}$. All photographs were exposed for equal times under identical conditions. Scale bar=200 $\mu \mathrm{m}$. 
A

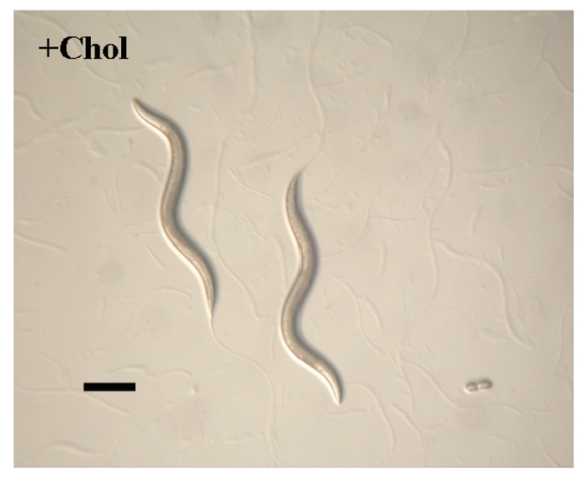

B

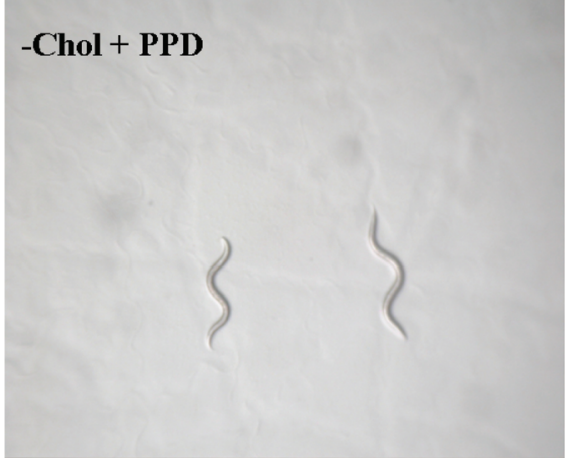

C

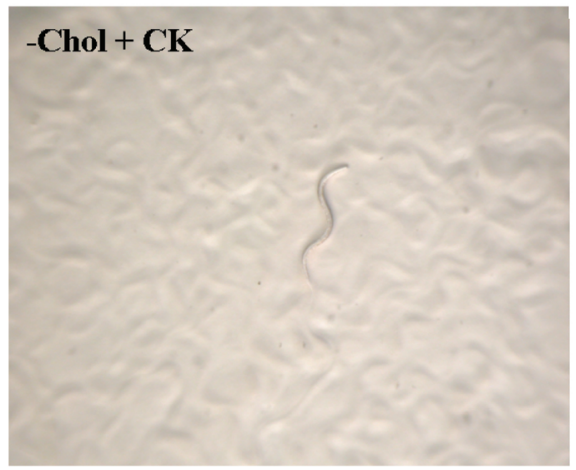

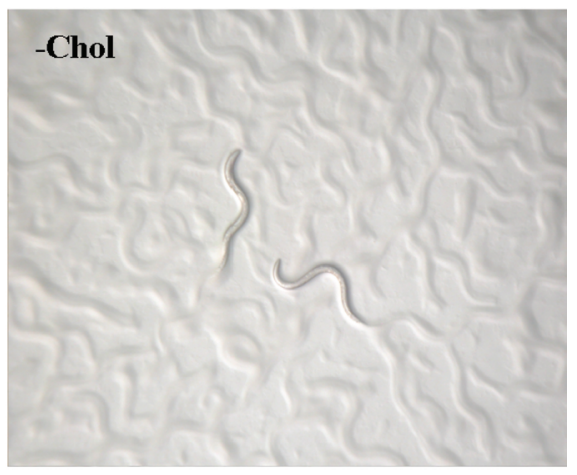

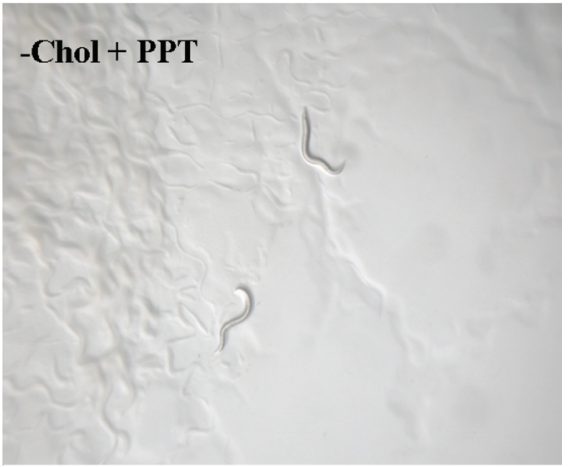

Fig. 3. Eggs laid by first generation animals were placed on plates containing the following concentrations: (A) cholesterol $5 \mathrm{mg} / \mathrm{mL}(+\mathrm{Chol})$, cholesterol-deprived (-Chol); (B) -Chol+300 $\mu \mathrm{M}$ protopanaxadiol (PPD), -Chol+300 $\mu \mathrm{M}$ protopanaxatriol (PPT); (C) -Chol+300 $\mu \mathrm{M}$ compound K (CK). Photographs were taken after $72 \mathrm{~h}$ growth of second generations at $20^{\circ} \mathrm{C}$. All photographs were exposed for equal times under identical conditions. Scale bar $=200 \mu \mathrm{m}$.

stage, respectively, and by 11, 24, and $27 \mathrm{~h}$ at L4, young adult, and adult stage in F2 generation, respectively, compared with worms grown in cholesterol-fed medium. In addition, we could observe the significant differences in the worm length between worms grown in cholesteroldeprived medium and worms grown in cholesterol-fed medium at L4, young adult, and adult stage (Figs. 2-4).

Supplement of $\mathrm{Rh}_{1}$ to cholesterol-deprived medium almost restored the growth rate and worm length in all stages of F1 generation (Table 1 and Fig. 4A). However, in F2 generation, supplement of $\mathrm{Rh}_{1}$ to cholesteroldeprived medium retarded the growth by 7,19 , and 24 at L4, young adult, and adult stage, respectively, compared with worms grown in cholesterol-fed medium (Table 1). In F2 generation worm length was slightly restored (Fig. 4B). Interestingly, supplement of $\mathrm{Rh}_{2}$ to cholesteroldeprived medium strongly suppressed worm growth, decreased worm size, and further retarded the worm growth by 7,20 , and $49 \mathrm{~h}$ at L4, young adult, and adult stage in F1 generation, respectively and by 7,19 , and $48 \mathrm{~h}$ at L4, young adult and adult in F2 generation, respectively, compared with worms grown in cholesterol-fed medium (Table 1), indicating that ginsenoside $\mathrm{Rh}_{1}$ or $\mathrm{Rh}_{2}$, which differs in the position of one carbohydrate, shows a differential effect on worm growth in cholesterol-deprived medium. 
A

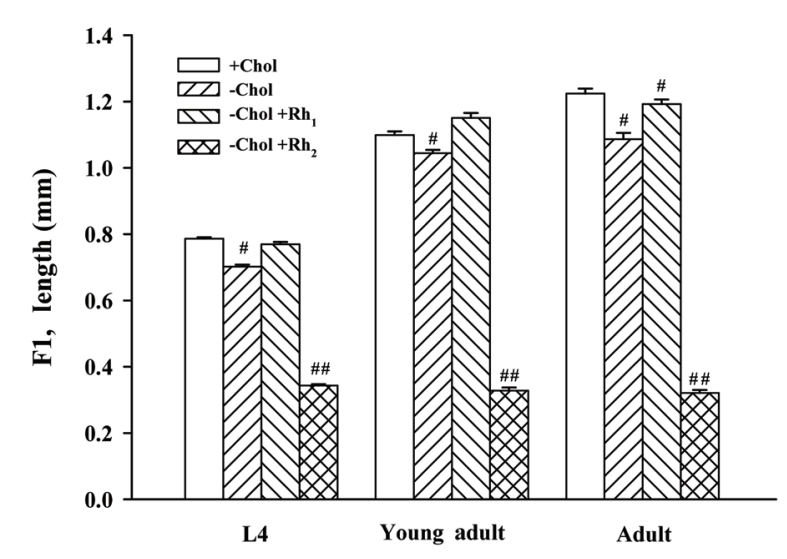

B

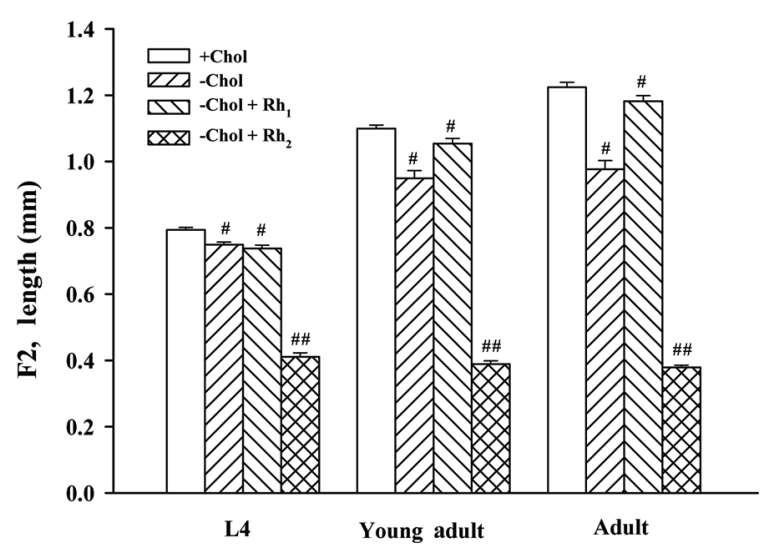

Fig. 4. Comparison of body size of worms treated with $300 \mu \mathrm{M}$ ginsenoside $R h_{1}$ or $300 \mu M$ ginsenoside $R h_{2}$. (A) In first generation (F1), specific stages (larvae 4 [L4], young adult, or adult) are indicated on the bottom of each bar with supplemented ginsenoside $\mathrm{Rh}_{1}$ or $\mathrm{Rh}_{2}$. The average value of twenty worms is presented. (B) In second generation (F2), specific stages (L4, young adult, or adult) are indicated on the bottom of each bar with supplemented ginsenoside $R h_{1}$ or $R h_{2}$. The average value of twenty worms is presented. Data are mean \pm SE. ${ }^{*} p<0.01$, different from cholesterol (Chol)-fed animals; ${ }^{\#} p<0.005$, different from Chol-fed animals.

\section{Effects of ginsenoside metabolites on worm growth in cholesterol-deprived medium}

We first examined the effects of compound K (CK) on the worm growth in cholesterol-deprived medium. Supplement of CK to cholesterol-deprived medium did not restore the growth rate rather further retarded the growth of worm retarded by 8,38 , and $43 \mathrm{~h}$ at L4, young adult and adult stage in F1 generation, respectively and by 25,43 , and $48 \mathrm{~h}$ at L4, young adult and adult in F2 generation, respectively, compared with worms grown in cholesterol-fed medium (Fig. 3C) and Table 1. Thus,
A

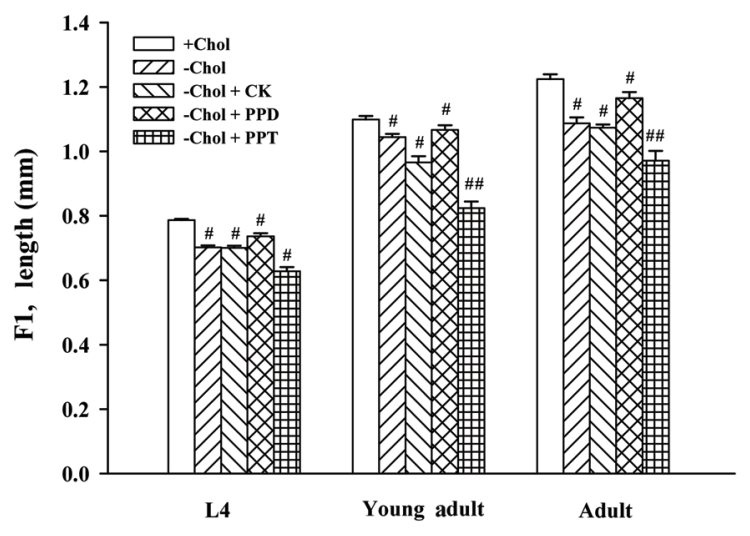

B

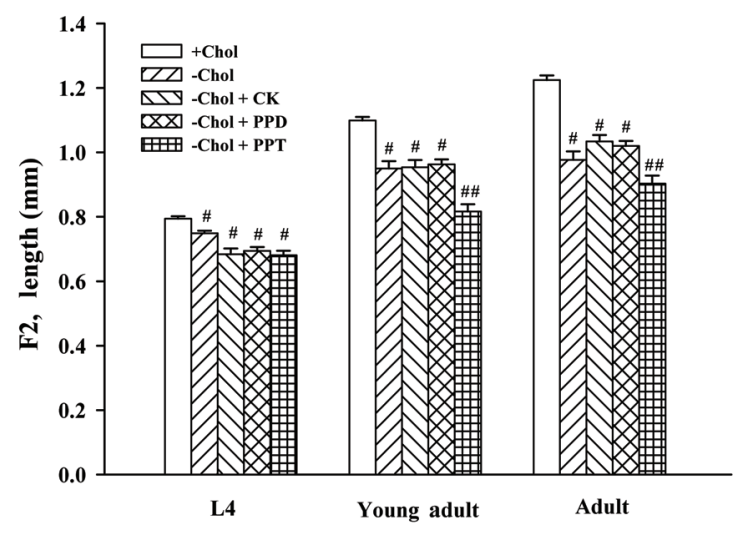

Fig. 5. Comparison of body size of worms treated with compound $\mathrm{K}$ (CK), 20(S)-protopanaxadiol (PPD), or 20(S)-protopanaxatriol (PPT) (300 $\mu \mathrm{M}$ each) in cholesterol-deprived medium. (A) The average value of twenty worms is presented. (B) In second generation (F2), specific stages are indicated on the bottom of each bar. The average value of twenty worms is presented. Data are mean \pm SE. F1, first generation; L4, larvae $4 .{ }^{*} p<0.01$, different from cholesterol (Chol)-fed animals; ${ }^{\#} p<0.005$, different from Chol-fed animals.

CK, which contains one carbohydrate at carbon-20, did not help much to restore worm growth in cholesteroldeprived condition. In addition, we could observe that supplement of CK to cholesterol-deprived medium did not restore the worm length throughout all stages of F1 and F2 and generations (Fig. 5).

Supplement of PPD to cholesterol-deprived medium did not restore worm growth and further retarded the worm growth by 15,36 , and $40 \mathrm{~h}$ at L4, young adult and adult stage in F1 generation, respectively and by 30,44 , and $48 \mathrm{~h}$ at L4, young adult and adult in F2 generation, respectively, compared with worms grown in cholesterolfed medium (Table 1 and Fig. 3B). Supplement of PPT to cholesterol-deprived medium further retarded the worm growth by 29,42 , and $46 \mathrm{~h}$ at L4, young adult, and adult 
A

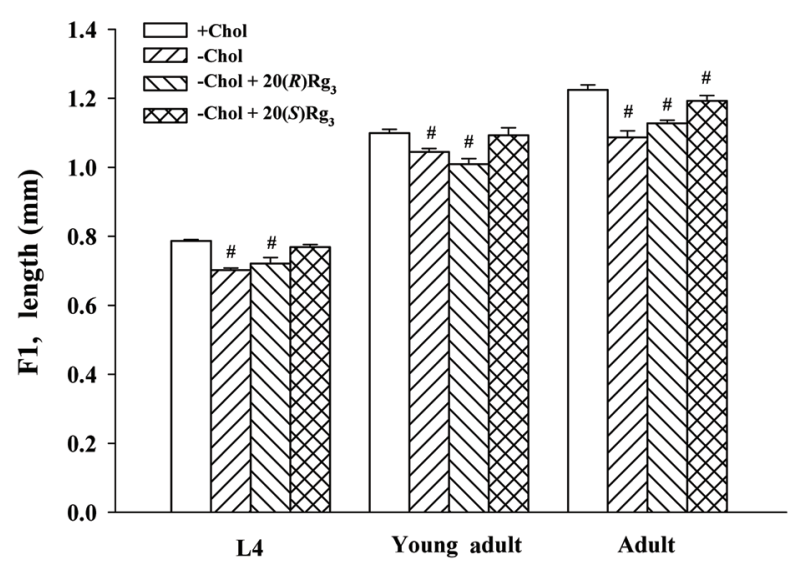

B

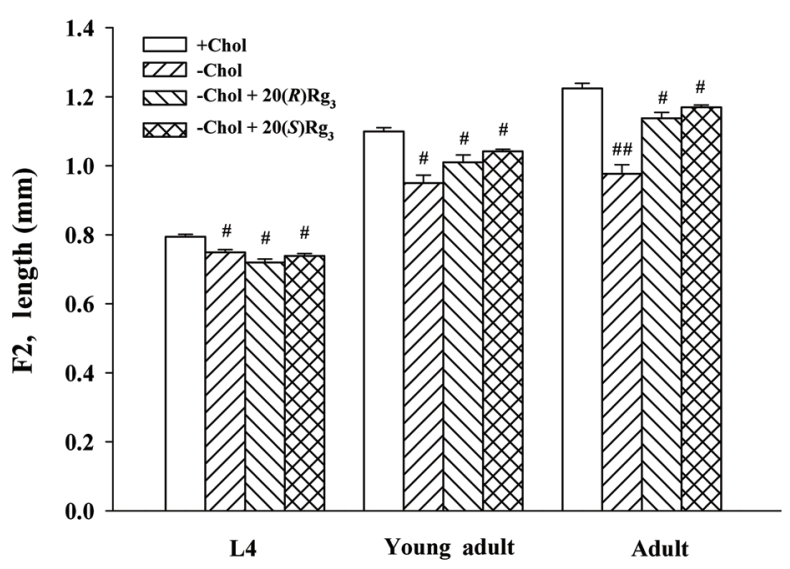

Fig. 6. Comparison of body size of worms treated with $300 \mu \mathrm{M}$ $20(R)-\mathrm{Rg}_{3}$ or $300 \mu \mathrm{M} 20(\mathrm{~S})-\mathrm{Rg}_{3}$ stereoisomers. (A) In first generation (F1), specific stages are indicated on the bottom of each bar. The average value of twenty worms is presented. (B) In second generation (F2), specific stages are indicated on the bottom of each bar. The average value of twenty worms is presented. Data are mean $\pm S E$. $\mathrm{L} 4$, larvae 4. ${ }^{\#} p<0.01$, different from cholesterol (Chol)-fed animals; ${ }^{\#} p<0.005$, different from Chol-fed animals.

stage in F1 generation, respectively and by 30,44 , and $48 \mathrm{~h}$ at L4, young adult, and adult in F2 generation, respectively, compared with worms grown in cholesterolfed medium (Table 1). Thus, supplement of PPD slightly restored worm length in cholesterol-deprived medium, whereas supplement of PPT to cholesterol-deprived medium rather retarded worm length in all stages of F1 and F2 generations (Fig. 5). These results indicate that the position of hydroxyl group of backbone of ginsenoside might also affect the worm growth in the cholesteroldeprived medium.

\section{Effects of ginsenoside epimers on worm growth in cholesterol-deprived medium}

Next, we examined the effects of ginsenoside epimers on worm growth in cholesterol-deprived medium. As shown in Table 1 and Fig. 2C, supplement of $20(R)-\mathrm{Rg}_{3}$ cholesterol-deprived medium retarded the worm growth by 8,19 , and $23 \mathrm{~h}$ at L4, young adult, and adult stage in F1 generation, respectively and by 8,19 , and $24 \mathrm{~h}$ at L4, young adult and adult in F2 generation, respectively, compared with worms grown in cholesterol-fed medium. In contrast, supplement of $20(S)-\mathrm{Rg}_{3}$ cholesterol-deprived medium almost restored worm growth and worm length was also restored at L4, young adult, and adult stage in F1 generation, respectively (Table 1, Figs. 2C and 6A) but in F2 generation supplement of $20(S)-\mathrm{Rg}_{3}$ cholesterol-deprived medium did not restore both worm growth and worm length as much as F1 generation compared with worms grown in cholesterol-fed medium (Table 1 and Fig. 6B). These results indicate that worms might have an ability to distinguish $20(S)-\mathrm{Rg}_{3}$ from $20(R)-\mathrm{Rg}_{3}$ for their normal growth in F1 and F2 generations.

\section{DISCUSSION}

Nematodes, including free-living $C$. elegans, require sterol for its normal development, growths, and life span as a nutritional source, since $C$. elegans is unable to biosynthesize sterol de novo [13]. However, C. elegans is usually able to obtain cholesterol or cholesterol-like sterols for their growth by metabolizing natural sterols such as phytosterols present in many plants or sterols from the animal body in the soil [14]. Although some species of nematodes are known to be parasitic in wild and cultivated ginseng roots [5], little is known on the physiological roles of ginseng saponins or ginsenosides to the reproduction, development, growth, and life span of $C$. elegans.

In the previous report, we could demonstrate that ginsenoside $R b_{1}$ and $R c$ but not $\mathrm{Rg}_{1}$ restored the average brood size, growth rate, percent development, and life span of C. elegans in cholesterol-deprived medium [6]. These results show a possibility that ginsenosides differentially affect development and growth of worm in cholesterol-deprived medium. In the present study, we examined the effects of minor ginsenosides, ginsenoside metabolites, or ginsenoside epimers on worm growth rate and worm length. We could observe that ginsenoside $\mathrm{Rh}_{1}$ and ginsenoside $\mathrm{Rh}_{2}$ exhibited a differential effect on worm growth in cholesterol-deprived medium. Supplement of ginsenoside $\mathrm{Rh}_{1}$ almost restored growth of worm 
compared with cholesterol-fed medium in F1 generation, whereas ginsenoside $\mathrm{Rh}_{2}$ exhibited deleterious effects by causing a death or arrest at embryos or larvae stage. We could not maintain worms until F3 generation (data not shown). Worms that even did hatch were also very sluggish, nearly paralyzed without movement. Interestingly, these two ginsenosides have only one carbohydrate on backbone structure but the position of carbohydrate is different from each other (Fig. 1). Ginsenoside $\mathrm{Rh}_{1}$ has a glucose at carbon 6 and ginsenoside $\mathrm{Rh}_{2}$ has a glucose at carbon-3. Thus, ginsenoside $\mathrm{Rh}_{2}$ could be utilized as anti-nematode agent (Fig. 4A, B). Taken together, these results indicate that the beneficial or harmful effects on $C$. elegans is depending on the type of ginsenosides, which is are different from the number and position of carbohydrates at carbon-3 or carbon- 6 and position of hydroxyl group at carbon 20.

In experiments using PPD and PPT, we could observe that although the time to reach at adult stage from egg was almost same between PPD and PPT in cholesteroldeprived medium, PPT produced smaller worms than those of PPD size but did not restore growth rate and worm size as much as cholesterol-fed medium in F1 and F2 generations (Fig. 4A). CK also delayed time to reach adult stage and did not restore worm size as much as cholesterol-fed medium (Fig. 5A, B).

In regard to structure-activity of ginsenoside $\mathrm{Rg}_{3}$ epimers, we have previously shown that $20(S)$ - but not $20(R)-\mathrm{Rg}_{3}$ inhibits voltage-dependent ion channel activities [7]. We also showed that in ex vivo experiments using swine coronary artery $20(S)$ - but not $20(R)-\operatorname{Rg}_{3}$ inhibited agonist-induced contraction of vessel [15]. These results indicate that $20(S)$ - rather than $20(R)-\mathrm{Rg}_{3}$ is a major active component for physiological or pharmacological actions. Furthermore, in the present in vivo study, we could demonstrate that supplement of $20(S)$ - but not $20(R)-\mathrm{Rg}_{3}$ to cholesterol-deprived medium almost restored worm growth rate and worm length as much as in cholesterol-fed medium. These results indicate that worms could distinguish $20(S)-\mathrm{Rg}_{3}$ from $20(R)-\mathrm{Rg}_{3}$, in which the position of hydroxyl group at carbon-20 differ, and utilize only $20(S)-\mathrm{Rg}_{3}$ for their growth under cholesterol-deprived condition. Again, we showed that 20(S)$\mathrm{Rg}_{3}$ but not $20(R)-\mathrm{Rg}_{3}$ could be biologically active.

In summary, using $C$. elegans as model system we herein used minor ginsenosides, ginsenoside metabolites, and ginsenoside epimers to know their roles in $C$. elegans growth in cholesterol-deprived-medium. We have obtained evidences that minor ginsenosides, ginsenoside metabolites, and ginsenoside epimers exhibit differential effects on C. elegans growth. Thus, ginsenoside 20(S)$\mathrm{Rg}_{3}$ and ginsenoside $\mathrm{Rh}_{1}$ could be used as a sterol substitute in cholesterol-deprived medium for C. elegans growth, whereas ginsenoside $\mathrm{Rh}_{2}$ could be used as an anti-nematode agent. Finally, these novel findings provide new insights that $C$. elegans could utilize subtypes of ginsenosides as sterol source.

\section{ACKNOWLEDGEMENTS}

This work was supported by Basic Science Research Program (2011-0021144) and the Priority Research Centers Program through the National Research Foundation of Korea (NRF), funded by the Ministry of Education, Science and Technology (2009-0093824), and by BK21 to SY Nah.

\section{REFERENCES}

1. Tyler VE. Herbal remedies. J Pharm Technol 1995; 11:214-220.

2. Hieb WF, Rothstein M. Sterol requirement for reproduction of a free-living nematode. Science 1968;160:778780.

3. Merris M, Wadsworth WG, Khamrai U, Bittman R, Chitwood DJ, Lenard J. Sterol effects and sites of sterol accumulation in Caenorhabditis elegans: developmental requirement for 4alpha-methyl sterols. J Lipid Res 2003;44:172-181.

4. Shim YH, Chun JH, Lee EY, Paik YK. Role of cholesterol in germ-line development of Caenorhabditis elegans. Mol Reprod Dev 2002;61:358-366.

5. Kim JH, Jeon YH, Park H, Lee BD, Cho DH, Park BY, Khan Z, Kim YH. The root-lesion nematode, Pratylenchus subpenetrans, on ginseng (Panax ginseng) in Korea. Nematology 2006;8:637-639.

6. Lee JH, Choi SH, Kwon OS, Shin TJ, Lee JH, Lee BH, Yoon IS, Pyo MK, Rhim H, Lim YH et al. Effects of ginsenosides, active ingredients of Panax ginseng, on development, growth, and life span of Caenorhabditis elegans. Biol Pharm Bull 2007;30:2126-2134.

7. Hasegawa H, Sung JH, Matsumiya S, Uchiyama M. Main ginseng saponin metabolites formed by intestinal bacteria. Planta Med 1996;62:453-457.

8. Jeong SM, Lee JH, Kim JH, Lee BH, Yoon IS, Lee JH, Kim DH, Rhim H, Kim Y, Nah SY. Stereospecificity of ginsenoside $\operatorname{Rg}_{3}$ action on ion channels. Mol Cells 2004; 18:383-389.

9. Tanaka O, Nagai M, Shibata S. Chemical studies on the oriental plant drugs. XVI. The stereochemistry of pro- 
topanaxadiol, a genuine sapogenin of ginseng. Chem Pharm Bull (Tokyo) 1966;14:1150-1156.

10. Shibata S, Tanaka O, Ando T, Sado M, Tsushima S, Ohsawa T. Chemical studies on oriental plant drugs. XIV. Protopanaxadiol, a genuine sapogenin of ginseng saponins. Chem Pharm Bull (Tokyo) 1966;14:595-600.

11. Brenner S. The genetics of Caenorhabditis elegans. Genetics 1974;77:71-94.

12. Matyash V, Entchev EV, Mende F, Wilsch-Brauninger M, Thiele C, Schmidt AW, Knolker HJ, Ward S, Kurzchalia TV. Sterol-derived hormone(s) controls entry into diapause in Caenorhabditis elegans by consecutive activation of DAF-12 and DAF-16. PLoS Biol 2004;2:e280.

13. Rothstein M. Nematode biochemistry. IX. Lack of sterol biosynthesis in free-living nematodes. Comp Biochem Physiol 1968;27:309-317.

14. Chitwood DJ, Lusby WR, Thompson MJ, Kochansky JP, Howarth OW. The glycosylceramides of the nematode Caenorhabditis elegans contain an unusual, branchedchain sphingoid base. Lipids 1995;30:567-573.

15. Kim JH, Lee JH, Jeong SM, Lee BH, Yoon IS, Lee JH, Choi SH, Kim DH, Park TK, Kim BK et al. Stereospecific effects of ginsenoside $\mathrm{Rg}_{3}$ epimers on swine coronary artery contractions. Biol Pharm Bull 2006;29:365-370. 